\title{
I ntersectorialidad en el contexto socioeconómico cubano y sus implicaciones en la salud de la población
}

\section{Intersectoral action in the Cuban socioeconomic context and its implications in the population health}

\author{
Dr. Cs. Pastor Castell-Florit Serrate, Dr. C. Estela de los Ángeles Gispert \\ Abreu
}

Escuela Nacional de Salud Pública. La Habana, Cuba.

\begin{abstract}
RESUMEN
El sistema de salud tiene como objetivo incrementar los niveles de salud, la calidad de los servicios, y la satisfacción de la población y de los prestadores. Para alcanzar estos propósitos, le concierne relacionarse, establecer alianzas e integrarse de manera sinérgica con otros sistemas de la sociedad y la economía a fin de ejercer control sobre los determinantes sociales de la salud. El propósito de este trabajo es identificar la percepción de actores sociales acerca del proceso de la intersectorialidad en su sector y sus implicaciones en la salud y el bienestar de la población. Se obtuvo información de 52 actores sociales clave en el contexto socioeconómico actual. Se pudo apreciar que a pesar de existir voluntad política y actores sociales convencidos, la realización del proceso de intersectorialidad para la salud y el bienestar en el contexto socioeconómico cubano, se percibe como baja, y que muchas de las situaciones que demandan intersectorialidad en la vida diaria demuestran insuficiente integración sectorial, ello indica que su aplicación en la actualidad no se corresponde con el alcance tecnológico que la intersectorialidad ha adquirido, por lo que hay necesidad de reforzar los elementos que competen al sector de la salud, y los vinculados a otros sectores de la sociedad y la economía.
\end{abstract}

Palabras clave: intersectorialidad, contexto social, salud.

\section{ABSTRACT}

The health system's objectives are to improve health status and quality of health services, as well as population and health worker satisfaction. To achieve this aims, it is important to establish alliances and act synergistically with other systems in society 
and the economy to affect the social determinants of health. This paper is based on research conducted in this year, aimed at identifying social actors' perceptions of the process of intersectoral action in their respective sectors and its implications for population health and wellbeing, with this purposed, information from 52 social key actors in Cuba's socioeconomic context were obtained. It was possible to realize that nonetheless, the existence of political will for intersectoral action in Cuba's context and of social actors persuaded of its relevance, current application of intersectoral action process is perceived as low and several situations demanded of intersectoral actions showed inadequate sectoral integration. This mean that intersectoral action is not as advanced as is technology in the health arena and manifest the necessity of strengthen, the elements in the health sector but also in those related to other areas of society and the economy.

Key words: intersectoral action, socioeconomic context, health.

\section{NTRODUCCI ÓN}

La intersectorialidad ha contribuido al afrontamiento de dificultades y a logros en el contexto socioeconómico cubano, por más de 50 años. El alcance de la intersectorialidad en Cuba no se ha debido a la espontaneidad sino que ha sido una concepción en correspondencia con la voluntad política del estado. ${ }^{1-3}$

Surgida de manera intuitiva, la intersectorialidad se desarrolló y consolidó en Cuba mediante el trabajo científico, primero como filosofía y como tecnología después, en el cursar de los años, brindando posibilidades para el perfeccionamiento de los procesos de gestión en el sector de la salud y buscando influencia en todos los sectores de la sociedad y la economía. En los sistemas y servicios de salud, ha sido un principio de la salud pública lo que ha favorecido su carácter integrador y la obtención de resultados de salud y bienestar.

Como tecnología, la intersectorialidad se basa en la aplicación del conocimiento científico a la solución de problemas prácticos a través de una cultura que provee métodos y medios para interactuar con el entorno social y físico, para así mejorar las condiciones de vida. Su concepción tecnológica en salud está dada por la generación de conocimientos, capacidades, destrezas técnicas, instrumentos y herramientas que se han generado a partir de los diferentes estudios e investigaciones, que posibilitan una mayor eficacia en la producción de salud. ${ }^{4}$

La intersectorialidad se enmarca dentro de los principios básicos conceptuales de una tecnología apropiada, ${ }^{4}$ porque forma parte de políticas y estrategias para el desarrollo sostenible de la salud a nivel nacional o internacional. También ofrece posibilidades para la formación de recursos humanos con valores éticos, y compromiso con una práctica profesional científica en función de la salud y el bienestar de la sociedad, influyendo en su disposición y utilización ante las demandas situacionales de salud y bienestar en su contexto.

La salud y el bienestar son producto de la confluencia de aspectos sociales, culturales, económicos, biológicos, ambientales, que conciernen a las familias, individuos, la comunidad y a los sectores sociales. Además de su valor intrínseco, la salud y el 
bienestar son imprescindibles para la realización personal y colectiva, por lo tanto, se asocian al éxito de una sociedad y sus instituciones de gobierno, lo que patentiza que no son patrimonio del sector de la salud y que para su fomento se requiere de políticas públicas intersectoriales a nivel global y local, ${ }^{5}$ respaldadas por la voluntad política de los Estados y sus gobiernos, que propicien la reflexión acción, retroalimentación colectiva, en torno a los factores que las condicionan, como parte de una gestión participativa, basada en el compromiso, el liderazgo y la toma de decisiones compartidas.

La necesidad de realizar las tareas de la salud pública con un enfoque interdisciplinario e intersectorial es innegable, ${ }^{6}$ pues la intersectorialidad proporciona la plataforma para el desarrollo de la acción integral por la salud y el bienestar en cualquier país o escenario, aunque difieran en el grado de desarrollo socioeconómico y humano, la historia, cultura, el ambiente natural, la estructura social, la organización, las funciones del gobierno y de cada sector en particular.

El sistema de salud tiene como objetivo incrementar los niveles de salud, la calidad de los servicios, y la satisfacción de la población y de los prestadores, convirtiéndose así en un componente del desarrollo social y en un instrumento de justicia social. Para alcanzar sus propósitos, le concierne relacionarse, establecer alianzas e integrarse de manera sinérgica con otros sistemas de la sociedad y la economía a fin de ejercer control sobre los determinantes sociales de la salud. ${ }^{7}$

Potenciar la cultura de la salud y la gestión eficiente de los servicios, haciendo prevalecer la promoción de salud y la prevención de riesgos ha sido imperativo de acción, que necesitan la intervención coordinada del sector de la salud con otros sectores socioeconómicos.

No obstante, existir voluntad política para la intersectorialidad en el contexto socioeconómico cubano y actores sociales convencidos de su pertinencia en materia de salud, su nivel de aplicación en la actualidad no se corresponde con el alcance tecnológico que la misma ha adquirido. Esto se manifiesta en que está menos universalizada, y sistematizada en su ejecución, no solo en los elementos que competen al sector de la salud, sino también en los vinculados a otros sectores de la sociedad y la economía.

En la medida que la intersectorialidad se conciba en la planeación estratégica de cada sector socioeconómico, se reincorpore a la cultura social, los diversos sectores dominen la disponibilidad y formas de aplicación de los instrumentos existentes y se retome la motivación de años precedentes, los resultados de acciones por la salud el bienestar y la calidad de vida serán cuantitativa y cualitativamente superiores.

La percepción de los actores sociales procedentes de varios sectores de la sociedad y la economía acerca de la intersectorialidad y sus implicaciones en la salud, es un reflejo bastante preciso de la misma en el contexto socioeconómico cubano y pudiera llevar a la práctica acciones que den respuesta real a los problemas que requieren intervención integrada

Los criterios emitidos en el presente trabajo se sustentan en una investigación realizada en el 2012, con el propósito de identificar la percepción de actores sociales acerca del proceso de la intersectorialidad en su sector, y las implicaciones de esta para la salud y el bienestar de la población. 


\section{MÉTODOS}

El diseño metodológico fue el de una investigación cualitativa cuantitativa, de corte transversal.

La información se obtuvo de 52 actores sociales considerados clave en el contexto socioeconómico actual, por estar vinculados a la dirección, a la docencia o a la práctica en el radio de acción de sus respectivos sectores: salud pública, recursos hidráulicos, comunicaciones, ciencia y tecnología y transporte; su elección fue por muestreo intencional dirigido a estudiantes de tres posgrados realizados en la Escuela Nacional de Salud Pública, que abordaron aspectos de actualidad para el contexto socioeconómico cubano; "Diplomado de Administración pública" en sus 2 ediciones y del curso "Cambio climático."

Los actores sociales encuestados se distribuyeron de la siguiente manera: sector de la salud 38

$(73,1 \%)$, otros sectores $14(26,9 \%)$, directivos $28(53,8 \%)$, no directivos 20 $(38,5 \%)$ y $4(7,7 \%)$ no respondieron.

El tiempo promedio en las funciones que realizaban fue de $12,4 \pm 10$ años, por lo que la mayoría eran directivos del sector de la salud y por el tiempo que tenían desempeñando su función, se pudiera decir que tienen conocimiento de su contexto.

Se exploraron variables valorativas, percepción de la realización del proceso de intersectorialidad en salud y bienestar (a través de la indagación en los aspectos, intersectorialidad misión visión, situaciones demandantes de participación intersectorial, fuentes de información intersectorial, sectores sociales involucrados, sectores con mayor responsabilidad, delimitación de la responsabilidad, recursos compartidos, control de las acciones, rendición de cuentas, acciones sobre la base de proyectos), y la percepción de las implicaciones de la intersectorialidad para la salud y el bienestar de la población (a través de los problemas de salud y bienestar apreciados como demandantes de intersectorialidad en su sector). Para ello, al inicio se exploró de manera grupal el conocimiento en el tema, "Intersectorialidad en la práctica social", identificándose deficiencias en cuanto a los factores de desarrollo de la intersectorialidad como tecnología; luego se impartió una conferencia de 45 min con igual título en el que se abordaron aspectos relacionados con la salud pública, las nuevas concepciones en salud, la intersectorialidad y su proceso y se entregó una encuesta estructurada de 10 preguntas. Se contó con el consentimiento de los participantes.

La encuesta (anexo) fue autoaplicada, y pretendía obtener información sobre la percepción de los actores sociales del proceso de intersectorialidad.

Se explicó que para su llenado se debía tener en cuenta la percepción personal de la intersectorialidad en su contexto sectorial. La encuesta se consideró como trabajo independiente y se recogió al día siguiente. Dentro de la clase se procedió al trabajo en equipo, para indagar acerca de cómo los actores sociales percibían las implicaciones de la intersectorialidad en salud. Cada grupo analizó en $30 \mathrm{~min}$ problemas de salud que demandaban participación intersectorial en su área de acción, un relator tomó nota y luego expuso en plenaria de forma resumida las opiniones del grupo, los demás participantes confrontaron sus experiencias e hicieron aportes, al finalizar el profesor presentó las conclusiones. El procesamiento de los resultados se realizó de froma computarizada.

A partir de los datos de la encuesta se calificó el nivel de realización del proceso de intersectorialidad como: alto, si 8 o más aspectos fueran adecuados al proceso de intersectorialidad; moderado, entre 6 y 7 aspectos adecuados y bajo, si 5 o menos 
aspectos eran adecuados. Las respuestas adecuadas se señalan en fuente itálica en el anexo.

\section{Anexo}

Encuesta acerca de la percepción de los procesos de intersectorialidad para la salud y el bienestar en Cuba

Estimado compañero (a):

La encuesta que sigue a continuación tiene como objetivo principal obtener información actualizada del estado de los procesos de intersectorialidad para la salud en su área de acción.

Este cuestionario es totalmente anónimo, por lo que puede responder con tranquilidad acerca de cuáles son sus impresiones, sentimientos o valoraciones reales sobre cada asunto preguntado, lo cual al mismo tiempo, representa una contribución importante de parte suya hacia el mejoramiento del sistema cubano de salud.

La Escuela Nacional de Salud Pública le agradece por anticipado su cooperación.

Sector al que pertenece: Función que realiza

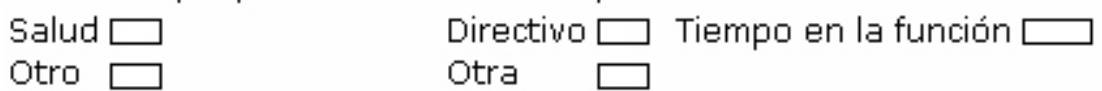

Por favor, en relación con el proceso de intersectorialidad para la salud en su área de acción o sector responda marcando con $\mathrm{X}$.

1. Concepción de la intersectorialidad para la salud (respuesta múltiple).

a) Concebida en la misión

b) Concebida en la visión

c) Cuando se indica por el nivel superior d) Ninguna de las anteriores e) No se tiene información al respecto

2. Situaciones que afectan la salud y el bienestar poblacional que demandan participación intersectorial (respuesta única).

a) Están identificados

b) En vías de su identificación

c) No están bien identificados

d) No se tiene información al respecto

3. Medios que se emplean para la obtención de la información acerca de las situaciones que afectan la salud y el bienestar poblacional (respuesta múltiple).

a) Análisis de la situación de salud

b) Otras investigaciones

c) Autopercepción

d) Percepción de la población sectores sociales f) Orientaciones del nivel superior información al respecto

e) Percepción de otros g) No se tiene

4. Sectores sociales involucrados en las situaciones que afectan la salud y el bienestar poblacional (respuesta única).

a) Están identificados

b) En vías de su identificación

http://scielo.sld.cu 
c) No están bien identificados

d) No se tiene información al respecto

5. Sectores de mayor responsabilidad en las situaciones que afectan la salud y el bienestar poblacional (respuesta única).

a) Están identificados

b) En vías de su identificación

c) No están bien identificados

d) No se tiene información al respecto

6. Planificación de acciones intersectoriales por la salud (AIS) en respuesta a los problemas que afectan la salud y el bienestar poblacional (respuesta única).

a) Están planificadas delimitando la responsabilidad de cada sector involucrado

b) Están planificadas sin delimitación de la responsabilidad de cada sector

involucrado____ c) Se ejecutan solo en casos de crisis, campañas o por orientaciones del nivel superior

d) No se tiene información al respecto

7. Tipo de recurso intersectorial con que cuenta habitualmente (respuesta múltiple).

a) Humanos

b) Materiales

c) Financieros

d) Ninguno de los anteriores

8. Control de las AIS (respuesta única).

a) Se monitorean y evalúan sistemáticamente por parte de todos los involucrados b) Se monitorean y evalúan sistemáticamente solo por parte del sector de la salud c) No se les da seguimiento

9. Divulgación del trabajo intersectorial en salud (respuesta única).

a) Rendición de cuentas sistemáticas

b) Rendición de cuentas ocasionales

c) Rendición de cuentas solo al sector de la salud habitualmente e) No se tiene información al respecto d) No se rinde cuentas

10. Proyectos u otras herramientas intersectoriales de salud. (respuesta única).

a) Se cuenta con proyectos en ejecución

b) Los que existen están detenidos c) No existen actualmente

d) Se desconoce la existencia de proyectos

La información procedente de la discusión grupal fue analizada, resumida y sintetizada según problema de salud y bienestar identificado como demandante de intersectorialidad.

\section{RESULTADOS}

Según los actores encuestados (tabla 1 ) la intersectorialidad estaba presente como parte de la misión y la visión de la mayoría de los sectores la sociedad y la economía, 
se identificaron en el 55,8 \% las situaciones que demandaban participación intersectorial, principalmente mediante el análisis de la situación de salud (84,6 \%), ya que el empleo de las otras fuentes como la percepción de otros sectores y de la comunidad fue insuficiente.

Tabla 1. Actores sociales según identificación del estado de los procesos de intersectorialidad $(n=52)$

\begin{tabular}{|c|c|c|}
\hline Procesos & No. & Porcentaje \\
\hline Concebida en la misión y visión & 36 & 69,2 \\
\hline Situaciones que demandan intersectorialidad & 29 & 55,8 \\
\hline Fuentes información & 2 & 3,8 \\
\hline Análisis de la situación de salud & 44 & 84,6 \\
\hline Fuentes de información intersectoriales & 17 & 32,7 \\
\hline Identificación de sectores sociales involucrados & 32 & 61,5 \\
\hline Sectores con mayor responsabilidad & 40 & 76,9 \\
\hline Delimitación de la responsabilidad de cada sector & 21 & 40,4 \\
\hline Recursos compartidos & 19 & 36,5 \\
\hline Monitoreo y evaluación sistemática & 20 & 38,5 \\
\hline Rendición de cuentas sistemáticas & 10 & 19,2 \\
\hline Proyectos de investigación & 26 & 50,0 \\
\hline Total & 52 & 100,0 \\
\hline
\end{tabular}

Los sectores sociales involucrados y los de mayor responsabilidad en la salud y el bienestar, son conocidos en la mayoría de los contextos $(61,5 \%$ y $76,9 \%)$, pero no todas las acciones intersectoriales son planificadas delimitando la responsabilidad de cada sector, ni se concibieron en proyectos, ello conjuntamente con las escasas rendiciones de cuentas $(19,2 \%)$, conspiraron contra la compartimentación de recursos humanos, materiales y financieros, así como contra el monitoreo y la evaluación intersectorial sistemática.

La realización del proceso de intersectorialidad para la salud y el bienestar en el contexto socioeconómico (tabla 2), se percibió como alto en solo el $17,3 \%$ de los sectores, prevaleció la calificación de baja sin resaltar diferencias según sector (tabla 3).

Tabla 2. Actores sociales según calificación del proceso de intersectorialidad

\begin{tabular}{|l|c|c|}
\hline Calificación & No. & $\%$ \\
\hline Alta & 9 & 17,3 \\
\hline Moderada & 13 & 25,0 \\
\hline Baja & 30 & $\mathbf{5 7 , 7}$ \\
\hline
\end{tabular}


Tabla 3. Actores sociales según sector y calificación del proceso de intersectorialidad

\begin{tabular}{|l|c|c|c|c|}
\hline \multirow{2}{*}{ Calificación } & \multicolumn{2}{|c|}{ Sector de la salud } & \multicolumn{2}{|c|}{ Otros } \\
\cline { 2 - 3 } & No. & $\%$ & No. & $\%$ \\
\hline Alta & 7 & 18,4 & 2 & 14,3 \\
\hline Moderada & 9 & 23,7 & 4 & 28,6 \\
\hline Baja & 22 & $\mathbf{5 7 , 9}$ & 8 & $\mathbf{5 7 , 1}$ \\
\hline Total & 38 & 100,0 & 14 & 100,0 \\
\hline
\end{tabular}

En cuanto a la percepción de los actores sociales acerca de las implicaciones de la intersectorialidad para la salud y el bienestar de la población se puso de manifiesto que:

- El trabajo por cuenta propia, (sector no estatal) se incorpora a la estructura socioeconómica del país de una manera acelerada con diversas formas de realización, lo que implica una clara delimitación de los sectores que con él se integran y de las formas de trabajo conjunto. Si tenemos en cuenta las dificultades que con frecuencia se aprecian en los centros gastronómicos y otros de expendio de alimentos estatales, en cuanto a las condiciones higiénicas y de otro tipo, esta nueva incorporación del sector no estatal deberá considerarse dentro del sistema de fiscalización, además de incrementar el rigor en las instalaciones del sector estatal.

- Situaciones tales como, las grasas utilizadas para la cocción que a veces superan las veces permisibles en su uso, el control de la existencia de agua en los establecimientos, la calidad de la higiene en los escenarios de los establecimientos, el uso de guantes y nasobuco por parte de los manipuladores de alimentos, son ejemplos que demandan la necesaria integración sectorial.

- Agromercados y expendedores de frutas, aceleran su proceso de maduración empleando químicos que dañan la salud y esto no se controla adecuadamente, cuando debiera existir un monitoreo y evaluación intersectorial sistemática.

- Los accidentes del tránsito están dentro de las principales causas de muerte en Cuba, las malas condiciones de las vías afectan a vehículos y transeúntes, en ocasiones su mal estado es consecuencia de trabajos de sectores tales como, recursos hidráulicos, comunicaciones, empresa eléctrica, por citar algunos, que realizan labores necesarias para el bienestar de la población, pero por otro lado crean afectaciones, cuando no son reparadas estas vías al concluir los trabajos inherentes a cada sector responsabilizado con ello. Es necesario la existencia de planes y programas conjuntos que de manera integrada intervengan en la solución de los problemas que existan con la delimitación de objetivos y acciones de cada cual.

- La comercialización y oferta de alimentos chatarra a la población merece un tratamiento diferenciado, estos crean hábitos alimenticios impropios, además de lo que representa para la salud el consumo de esos productos con afectación de cierto sector poblacional y en particular aquellos que son más consumidos por la población infantil, lo que demanda que los integrantes de estructuras organizativas de carácter intersectorial evalúen a profundidad estos problemas y surjan propuestas de solución Es significativo que con frecuencia estas ofertas se realizan en el entorno de instalaciones de salud preferentemente hospitales. 
- El control de vectores, actividad rectorada por el sector de la salud, es un ejemplo fehaciente de responsabilidad colectiva, que requiere niveles de integración sectorial diversos. Un porcentaje importante de sectores de la sociedad y la economía están implicados en ello, locales cerrados, salideros de agua, basuras y escombros en lugares inapropiados, es un ejemplo de situaciones palpables generadora de vectores

- El deterioro paulatino de la calidad en la prestación de los servicios, y conductas impropias por un grupo no despreciable de personas, es una situación problema de carácter intersectorial; en ello inciden diversos factores: indisciplinas, aspectos de comportamiento y degradación de valores, donde el factor multicausalidad efecto, está latente, pero basado en el concepto tan acostumbrado, de responsabilidad de todos, no posibilita a veces, tener en cuenta la responsabilidad individual, lo que trae como consecuencia que las soluciones, o no se aprecian, no respondan a la realidad del problema o se dilatan innecesariamente.

- El tabaquismo continúa siendo un problema importante y se está observando desde edades tempranas, algunos establecimientos no respetan la prohibición de su venta a menores, también existe indolencia de varios fumadores que no fuman en las áreas disponibles para ello, últimamente se ven fumadores en los ómnibus y cines, también hay madres y padres que fuman en presencia de sus hijos, fumadores pasivos. Para el control de esta adicción es necesario que los actores de los sectores de la sociedad cumplan y hagan cumplir las legislaciones al efecto. La ejemplaridad de profesionales y trabajadores de la salud en general, así como los maestros que fuman deliberadamente en ocasiones, deberá ser una premisa. La publicidad en los medios de divulgación debe dirigirse a desestimular este hábito, no ha favorecerlo.

- El embarazo en la adolescencia está aumentando en varias regiones, esto requiere de un trabajo mancomunado de los sectores de educación, salud, la federación de mujeres cubanas, y los medios de difusión masiva en otros sectores, principalmente con las adolescentes y sus familias, pero también con los adolescentes varones y sus familias ya que la responsabilidad es de ambos sexos.

- La ingestión de bebidas alcohólicas es frecuente y varios jóvenes muestran adicción ante las limitadas opciones recreativas. El trabajo integrado de sectores posibilitaría crear opciones para los jóvenes y controlar el expendio de alcohol de una manera inteligente no mecánicamente restrictiva.

- La población cubana está envejeciendo por lo que es necesario incrementar la atención a la tercera edad, en ocasiones adultos mayores presentan enfermedades crónicas y discapacitantes y existen ciertas dificultades con la atención médica integrada, pero además con la oferta de sillones de rueda y bastones, así como barreras arquitectónicas dentro de los servicios de salud y otros escenarios. Por otra parte, algunas personas en edad laboral tienen que abandonar su trabajo para fungir como cuidadores, para estos aspectos urgen estrategias intersectoriales

- Los círculos y las casas de abuelos necesitan atención de los sectores de la comunidad para diversificar las opciones e incrementar las actividades propias de la tercera edad.

\section{DISCUSIÓN}

Las situaciones referidas que demandan intersectorialidad se aprecian en la vida diaria y demuestran insuficiente integración sectorial por lo que hay necesidad de reforzar las alianzas y enfoques intersectoriales.

En Cuba, luego de 1959, las políticas públicas saludables se han encaminado a garantizar la "salud para todos" a través del desarrollo de estrategias integrales con 
voluntad política y acción intersectorial a partir de la identificación de los determinantes sociales de cada momento. $3,8,9$

En la actualidad el contexto socioeconómico ha venido transformándose en respuesta a los lineamientos de la política económica y social del Partido, ${ }^{10}$ que tienen como fundamento la batalla económica como tarea principal, en este sentido se requieren enfoques intersectoriales en correspondencia con la producción social de salud ${ }^{11}$ a través de lo cual se lograría un acercamiento a la solución de los problemas mencionados, entonces así se mejorarían los resultados en el estado de salud poblacional y en el bienestar poblacional, complementariamente se disminuirían los gastos en salud como consecuencia del elevado costo de medicamentos y el proceso de hospitalización, este último como problema adicional implica perdidas de horas laborales y escolares y repercute significativamente en la economía personal y social.

En resumen, las transformaciones puestas en práctica en el sector de la salud están encaminadas a lograr mayor eficiencia, eficacia y efectividad, lo que determina formas de relaciones ajustadas al contexto social y económico del presente y lleva a un mayor nivel de influencia del sector de la salud con los demás sectores sociales, incluyendo el sector no estatal, que se incorpora aceleradamente a la economía nacional. La investigación realizada reveló fallas en la implementación tecnológica de la intersectorialidad, en cuanto al cumplimiento de las leyes, regulaciones, directivas y otras disposiciones que establecen el trabajo integrado para accionar ante los problemas descritos; su fortalecimiento se impone para que las estrategias por la salud y el bienestar cumplan su cometido.

La percepción de los actores sociales procedentes de varios sectores de la sociedad y la economía acerca de la intersectorialidad y sus implicaciones en la salud, se corresponde con su limitada aplicación y el no aprovechamiento de las posibilidades que brinda esta aplicación como tecnología ante la situación actual en el contexto socioeconómico cubano.

\section{Agradecimientos}

A los profesores de la Escuela Nacional de salud Pública: Lic. Anabel Lozano Lefrán, Dra. Lourdes Couturejuzón González, Dr. C. Orlando Carnota Lauzán y Dra.

Magdalena Mirabal J ean Claude, por su contribución.

\section{REFERENCI AS BI BLI OGRÁFICAS}

1. Castell-Florit Serrate P. La intersectorialidad en la práctica social. La Habana: Editorial Ciencias Médicas; 2007.

2. Castell-Florit Serrate P, Gispert Abreu, E de los Á. La intersectorialidad y el desarrollo de la Salud Pública en Cuba. Rev Cubana Salud Pública [Internet línea]. 2009 [citado 5 Abr 2008]; 35(1). Disponible en:

http://scielo.sld.cu/scielo.php?script=sci_arttext\&pid=S0864-

34662009000100004\&lng=es

3. Álvarez Pérez AG, García Fariñas A, Rodríguez Salvá A, Bonet Gorbea M. Voluntad política y acción intersectorial: Premisas clave para la determinación social de la salud en Cuba. Rev Cubana Hig Epidemiol [Internet]. 2007 [citado 8 Ago 2012]; 45(3).

http://scielo.sld.cu 
Disponible en: http://scielo.sld.cu/scielo.php?script=sci_arttext\&pid=S1561$30032007000300007 \& \operatorname{lng}=\mathrm{es}$

4. Castell-Florit Serrate P. La intersectorialidad, una tecnología que despega con fuerza. Rev Cubana Salud Pública [Internet]. 2010 [citado 6 Ago 2012];36(1): 101-2. Disponible en: http://scielo. sld.cu/pdf/rcsp/v36n2/spu01210.pdf

5. CastelL-Florit Serrate P, Carnota Lauzán O, Mirabal J ean-Claude M, Santana Espinosa MC, Cabrera González T, Hadad Hadad J, et al. Intersectorialidad en Cuba, su expresión a nivel global y local. La Habana: Editorial Ciencias Médicas; 2008.

6. Rojas Ochoa F. Interdisciplina e Intersectorialidad. Rev Cubana Salud Pública [Internet]. 2010 [citado 6 Ago 2012];36(3). Disponible en: http://scielo.sld.cu/pdf/rcsp/v36n3/spu01310.pdf

7. Castell-Florit P. Intersectorialidad y sistemas de salud. La experiencia cubana [Internet]. Ministerio de Salud Pública: ENSAP; 2003 [citado 5 Abr 2008]. Disponible en: http://www.sld.cu/galerias/doc/sitios/infodir /20 intersectorialidad.doc

8. CastelL-Florit Serrate P. Comprensión conceptual y factores que intervienen en el desarrollo de la intersectorialidad. Rev Cubana Salud Pública [Internet]. 2007 [citado 6 Ago 2012];33(2). Disponible en:

http://bvs.sld.cu/revistas/spu/vol33_02_07/spu09207.htm

9. Castell-Florit Serrate P, Gispert Abreu E de los Á. El Sistema Nacional de Salud cubano y su orientación hacia el perfeccionamiento continuo [I nternet]. 2011[citado 5 Abr 2008]. Disponible en:

http://fnsp.udea.edu.co/fnsp/congresosp7/PCastellFlorit.pdf

10. Lineamientos de la política económica y social del Partido y la Revolución [Internet]. 2011 [citado 25 May 2012]. Disponible en: http://www.cubadebate.cu/wpcontent/uploads/2011/05/folleto-lineamientos-vi -cong.pdf

11. Castell-Florit Serrate P. Intersectoral Health Strategies: From Discourse to Action [Internet]. 2010 [cited 2012 May 25]; 12(1): 48. Available from:

http://www. medicc.org/mediccreview/articles/mr_134.pdf

Pastor Castell-Florit Serrate. Escuela Nacional de Salud Pública. Calle 100 No. 1132 e/ E y Perla. Altahabana, municipio Boyeros. La Habana, Cuba.

Correo electrónico: pastor@infomed.sld.cu 